\title{
Defoliación en la especie invierno-verde Aristotelia chilensis y su efecto sobre el crecimiento inicial de hojas y ramas
}

\author{
Defoliation in the evergreen species Aristotelia chilensis and its effect on initial \\ foliar and branch growth
}

\author{
MARIA ANGELICA DAMASCOS ${ }^{1}$, CARLOS HENRIQUE B.A. PRADO ${ }^{2}$ \\ ${ }^{1}$ Departamento de Ecología. Universidad Nacional del Comahue, Unidad Postal Universidad. 8400 \\ San Carlos de Bariloche, Argentina. ${ }^{2}$ Departamento de Botánica, \\ Universidade Federal de São Carlos, CP 676, Cep: 13565-905, São Carlos, SP, Brasil.
}

\section{SUMMARY}

Defoliation effects on vegetative growth in early spring were evaluated in the evergreen species Aristotelia chilensis growing in two areas of Nothofagus dombeyi forest in Argentina. In both studied sites (S1 and S2) the mean values of the linear initial branch growth were lower and statistically different in defoliated plants (S1: 2,48 $\pm 0.98 \mathrm{~cm}, \mathrm{~S} 2$ : $2,08 \pm 0,77 \mathrm{~cm}$ ) than in control plants $(\mathrm{S} 1: 6,13 \pm 4,88 \mathrm{~cm}, \mathrm{~S} 2: 6,38 \pm 0,69 \mathrm{~cm})$. However, new leaves flushed in early

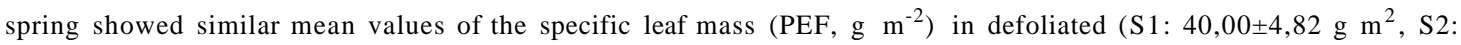
$52,10 \pm 18,10 \mathrm{~g} \mathrm{~m}^{-2}$ ) and in control plants $\left(\mathrm{S} 1: 42,10 \pm 20,48 \mathrm{~g} \mathrm{~m}^{-2}, \mathrm{~S} 2: 48,5 \pm 16,4 \mathrm{~g} \mathrm{~m}^{-2}\right)$. The results are related to short and long term whole plant carbon balance. Defoliated plants would be affected in their short term carbon balance and they would not have resources to maintain the branch linear growth (a carbon sink). Defoliated plants sustained the construction of the new leaves (a carbon source when expanded) in order to assure the long term carbon balance as positive as possible.

Key words: Aristotelia chilensis, evergreen species, growth, specific leaf mass, experimental defoliation.

\section{RESUMEN}

Los efectos de la defoliación sobre el crecimiento vegetativo a principios de primavera fueron evaluados en la especie siempreverde Aristotelia chilensis, creciendo en dos áreas del bosque de Nothofagus dombeyi, en Argentina. En ambos sitios estudiados (S1 y S2) los valores medios de crecimiento lineal inicial de los brotes fueron menores y estadísticamente diferentes en las plantas defoliadas ( $\mathrm{S} 1: 2,48 \pm 0,98 \mathrm{~cm}, \mathrm{~S} 2: 2,08 \pm 0,77 \mathrm{~cm})$ que en los controles

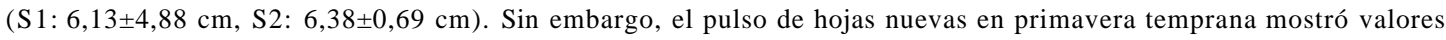
medios similares de peso específico foliar (PEF, $\mathrm{g} \mathrm{m}^{-2}$ ) en las plantas defoliadas ( $\mathrm{S} 1: 40,00 \pm 4,82 \mathrm{~g} \mathrm{~m}^{-2}, \mathrm{~S} 2: 52,10 \pm 18,10$ $\mathrm{g} \mathrm{m}^{-2}$ ) y en los controles ( $\mathrm{S} 1: 42,10 \pm 20,48 \mathrm{~g} \mathrm{~m}^{-2} ; \mathrm{S} 2: 48,5 \pm 16,4 \mathrm{~g} \mathrm{~m}^{-2}$ ). Los resultados están relacionados con el balance de carbono de la planta a corto y largo plazo. Las plantas defoliadas podrían ser afectadas en su balance de carbono a corto plazo y estas no tendrían recursos para mantener el crecimiento lineal de los brotes (sumideros de carbono). Por otro lado, las plantas defoliadas sustentaron la construcción de las nuevas hojas (fuente de carbono cuando están expandidas) en orden de asegurar el balance de carbono a largo plazo lo más positivo posible.

Palabras claves: Aristotelia chilensis, especie siempreverde, crecimiento, peso específico foliar, defoliación experimental. 


\section{INTRODUCCION}

Las especies siempreverdes (Bell y Bliss, 1977) conservan sus hojas sólo durante un invierno, siendo las mismas fotosintéticamente activas tanto en los días de temperatura moderada de otoño e invierno (Landhäusser et al., 1997; Minoletti y Boerner, 1993) como durante la primavera temprana (Tissue et al., 1995; Prado y Damascos, en prensa). Jonasson (1995) indicó que en el arbusto rastrero de fenología foliar invierno-verde Rhododendron lapponicum, la principal función de la retención invernal de la cohorte de hojas viejas era mejorar el balance de carbono de la planta. En esta especie la eliminación de dichas hojas en primavera temprana afectó negativamente la biomasa de ramas, pero no causó cambios significativos en la concentración de nutrientes de las hojas formadas en primavera. La defoliación de ramas individuales en la misma especie se reflejó en una menor área foliar y peso, tanto de las hojas formadas en primavera como de las ramas y de los órganos reproductivos (Karlsson, 1994). Luego de la eliminación de las hojas viejas de la orquídea invierno-verde Tipularia discolor, las plantas no se reprodujeron sexualmente y sufrieron una disminución de la biomasa de sus órganos subterráneos (Whigham, 1990).

Aristotelia chilensis (Mol.) Stuntz (Elaeocarpaceae) especie arbustiva nativa de los bosques andino-patagónicos que crece tanto en áreas abiertas como debajo del dosel de los bosques (Damascos, 1998) posee una fenología foliar invierno-verde. Sus hojas emergen en dos períodos, un grupo se produce en forma de pulso a principios de primavera, mientras que durante el verano emergen otras hojas en forma secuencial. Una parte de estas hojas, principalmente las de la cohorte formada en primavera, senesce en otoño, mientras que la totalidad de las hojas remanentes cae en la siguiente primavera un mes después de la expansión de la cohorte de hojas nuevas (Damascos y Prado ${ }^{1}$ ). El estudio de esta planta es importante, porque es una especie de alta frecuencia en los bosques andino-patagónicos (Damascos, 1996) y pertenece a un grupo fenológico no estudiado en plantas del hemisferio Sur. En un trabajo previo (Damascos y

1 DAMASCOS, M. A. y C. H. PRADO. 2001. Fenología foliar y sus caracteres asociados en la especie "wintergreen" Aristotelia chilensis. Informe Interno, Universidad Nacional del Comahue.
Prado $^{1}$ ) se analizó la fenología de A. chilensis y se comparó su peso específico foliar (PEF, $\mathrm{g} \mathrm{m}^{-2}$ ) con el de las hojas de especies leñosas deciduas y siempreverdes de los mismos bosques y de especies de comunidades de otras regiones. Por otro lado, se comprobó que las hojas de esta especie, que sobreviven durante todo el invierno ("hojas viejas"), son fotosintéticamente activas durante la primavera y 15 días antes de la senescencia, sometidas a un bajo flujo fotónico $\left(150 \mathrm{~m} \mathrm{~mol} \mathrm{~m}^{-2} \mathrm{~s}^{-1}\right)$ muestran mayores valores medios de fotosíntesis y de eficiencia en el uso del agua expresados en área que las hojas nuevas (Prado y Damascos, en prensa). Se desconoce cuál es el efecto de la ausencia de las hojas viejas de esta especie sobre el crecimiento vegetativo de la planta en primavera.

En este trabajo se evalúan las consecuencias de la eliminación total de las hojas viejas de $A$. chilensis a fines de invierno, sobre el crecimiento en primavera de los nuevos brotes y el peso específico de las hojas nuevas. Se hipotetiza que la conservación por un invierno de las hojas viejas, en esta especie, tiene principalmente como objeto servir de fuente de carbono para la formación del pulso primaveral de hojas nuevas y para el crecimiento de las ramas.

\section{MATERIALES Y METODOS}

Se estudiaron plantas de A. chilensis ("maqui") del bosque perennifolio de Nothofagus dombeyi (Fagaceae) presentes en el área de Llao-Llao, cercana a la ciudad de San Carlos de Bariloche $\left(41^{\circ} 04^{\prime} \mathrm{S}-71^{\circ} 08^{\prime} \mathrm{W}\right)$ en Argentina. La precipitación media anual en el área de estudio es de 2.000-2.200 mm (Barros et al., 1983) y está concentrada en los meses de otoño e invierno. Durante 1998 la temperatura media fue de $5,8^{\circ} \mathrm{C}$ en julio, $5,5^{\circ} \mathrm{C}$ en septiembre y $14,4^{\circ} \mathrm{C}$ en diciembre.

Se eligieron dos sitios cercanos (separados por $300 \mathrm{~m}$ ) ubicados a $1.100 \mathrm{~m}$ de altitud, en un área con relieve plano y con condiciones ambientales similares dentro de un bosque maduro de $N$. dombeyi. En cada sitio se eligieron 10 plantas de A. chilensis, de las cuales cinco plantas por sitio fueron totalmente defoliadas de sus hojas viejas a fines del invierno, antes de la apertura de las yemas vegetativas y florales, mientras que otras cinco plantas por sitio fueron dejadas como control.

En uno de los sitios a mediados de noviembre se eligieron al azar otras 10 plantas de A. chilensis, 
las que fueron usadas para determinar el peso seco total y estimar el área foliar total por rama de las cohortes de hojas viejas y nuevas presentes sobre las mismas en primavera y comparar el grado de expansión de las hojas nuevas antes de la senescencia de las hojas viejas.

Todas las plantas incluidas en este estudio tenían más de un metro de altura, estaban ramificadas y crecían en áreas abiertas del bosque.

A fin de evitar la posible compensación de la falta de las hojas viejas a partir de reservas preexistentes en la planta y de la actividad de las hojas nuevas formadas a principios de primavera, se estudió sólo el crecimiento inicial de los nuevos brotes formados en las plantas defoliadas y en los controles. El 4 de noviembre de 1998, 50 días después de realizada la defoliación de las hojas viejas, todos los nuevos brotes del pulso de primavera, recientemente formados sobre la planta e identificables por su color verde claro, fueron cortados tanto de las plantas defoliadas (5 plantas por sitio) como de los controles (5 plantas por sitio). El número total de brotes por planta varió entre 10 y 50 en las plantas defoliadas y 10 y 35 en los controles. Se midió la longitud de cada brote y se separaron todas las nuevas hojas presentes en cada uno. Muestras de 50 hojas nuevas por planta, sin síntomas de predación o enfermedad, fueron usadas para la determinación del peso específico foliar (PEF, g. $\mathrm{m}^{-2}$ ). El muestreo y determinación del PEF se realizó siguiendo el protocolo recomendado por Westoby (1998). En cada hoja y en una posición alejada de las nervaduras principales, se cortó con un sacabocados un disco foliar. Los discos fueron secados en estufa a $60^{\circ} \mathrm{C}$ hasta peso constante y pesados. Usando los valores de área y peso total de los discos foliares de cada muestra se calculó el PEF para cada planta.

Por otro lado, a mediados de noviembre se cortó una rama de cada una de 10 plantas (no consideradas en el experimento de defoliación) y se colectaron todas las hojas viejas y nuevas de la rama, las cuales fueron secadas en estufa hasta peso constante. Con el peso total y el PEF de "hojas viejas" y nuevas (obtenido con la metodología antes descrita) se calculó el área fotosintética total de cada tipo de hoja por rama. Para analizar el grado de expansión de las hojas nuevas en los días previos a la caída de las hojas viejas, se midió además la longitud de 150 hojas viejas y 150 hojas nuevas.

El alargamiento inicial de los brotes y el PEF de la hojas nuevas fueron estudiados en forma separada mediante un análisis de varianza de doble entrada (Sokal y Rohlf, 1981) con dos factores: tratamiento (defoliada y control) y sitio (1 y 2).

Tanto el peso total como el área foliar total por rama de las cohortes de hojas viejas y nuevas se compararon usando la prueba de $t$ de Student para muestras dependientes, mientras que para comparar la longitud de 150 hojas de cada tipo, y el PEF de las hojas viejas y nuevas en primavera se usó la misma prueba, pero para muestras independientes (Sokal y Rohlf, 1981).

\section{RESULTADOS Y DISCUSION}

Aunque una parte importante de las hojas de A. chilensis formadas en el primer pulso de emergencia foliar de primavera cae durante el otoño, una porción de estas hojas, junto con las formadas durante el verano, sobrevive durante el invierno (Damascos y Prado ${ }^{1}$ ). Estas hojas viejas pierden masa foliar durante la estación de reposo, pero en la siguiente primavera son fotosintéticamente activas (Prado y Damascos, en prensa), su área fotosintética total por rama es semejante a la de la nueva cohorte foliar de primavera, pero su masa foliar total por rama es mayor (cuadro 1). Por tanto, su contribución al balance de carbono de la planta sería significativo durante este período.

La figura 1 muestra que si la cohorte de hojas viejas de A. chilensis está ausente de la planta a principios de primavera, los nuevos brotes crecen menos en relación a los de las plantas que no fueron defoliadas, siendo esta diferencia independiente del sitio estudiado (cuadro 2, fig. 1). Estos brotes recientemente formados constituyen sumideros de masa y energía requiriendo recursos tanto para su crecimiento y mantenimiento como para la formación de la nueva cohorte foliar de primavera y para la floración. La formación y crecimiento de las estructuras reproductivas de la planta son procesos con alta demanda de energía (Snow y Whigham, 1989) y las hojas nuevas, por su parte, son costosas en términos de construcción, porque al ser más tiernas requieren de más compuestos de defensa contra la herbiboría (Bazzaz et al. 1987).

En otros estudios realizados en especies con fenología foliar invierno-verde se encontró que la conservación de las hojas viejas hasta la siguiente primavera no estaba asociada a la retraslocación de nutrientes foliares antes de la formación de las hojas nuevas, pero sí al mantenimiento de un ba- 


\section{CUADRO 1}

Valores medios \pm desviación standard del peso seco foliar total por rama, área foliar total por rama, longitud de la hoja y peso específico foliar (PEF) de las cohortes de hojas viejas y nuevas en la especie inviernoverde Aristotelia chilensis a principios de primavera (15 días antes de la caída total de la cohorte de hojas viejas). Medias seguidas de las mismas letras indican ausencia de diferencias estadísticas significativas entre hojas viejas y nuevas $(\mathrm{p}<0,05)$.

Mean values \pm standard deviation of the leaf total dry mass per branch, total leaf area per branch, leaf length, and the specific leaf mass (PEF) of the old and new leaf cohorts in a wintergreen species Aristotelia chilensis at the beginning of the spring (fifty days before the total fall of the old cohort). Means followed by same letters indicate absence of significant statistical differences between old and new leaves $(\mathrm{p}<0,05)$.

\begin{tabular}{|c|c|c|}
\hline & \multicolumn{2}{|c|}{ Hojas } \\
\hline & Viejas & Nuevas \\
\hline Peso seco total por rama $(\mathrm{g}), \mathrm{n}=10$ ramas & $4,65 \pm 2,90 \mathrm{a}$ & $1,73 \pm 0,95 \mathrm{~b}$ \\
\hline Area foliar total por rama $\left(\mathrm{cm}^{2}\right), \mathrm{n}=10$ ramas & $552,03 \pm 345,15 \mathrm{a}$ & $410,81 \pm 224,63 \mathrm{a}$ \\
\hline Longitud de la hoja $(\mathrm{cm}), \mathrm{n}=150$ hojas & $8,85 \pm \pm 1,71$ a & $7,69 \pm 1,71 \mathrm{a}$ \\
\hline $\operatorname{PEF}\left(\mathrm{g} \cdot \mathrm{m}^{-2}\right), \mathrm{n}=5$ plantas & $80,56 \pm 11,62 \mathrm{a}$ & $45,86 \pm 14,38 \mathrm{~b}$ \\
\hline
\end{tabular}

\section{CUADRO 2}

Efecto de los tratamientos (defoliación y control) de los sitios e interacción entre sitios y tratamientos sobre el crecimiento inicial lineal de los brotes y el peso específico foliar (PEF, $\mathrm{g} \cdot \mathrm{m}^{-2}$ ) en la especie inviernoverde Aristotelia chilensis creciendo en dos sitios del bosque de Nothofagus dombeyi.

(Análisis de varianza de dos vías, GL: grados de libertad, CM: cuadrado medio,

$F$ : estadístico de Fisher, $P$ : valor de significación).

Effect of the treatments (defoliation and control), the sites, and the interaction between sites and treatments on the initial linear branch growth and the specific leaf mass (PEF, g. $\mathrm{m}^{-2}$ ) in a wintergreen species Aristotelia chilensis growing in two sites at Nothofagus dombeyi forest. (Two way-analysis of variance, $G L$ : degrees of freedom, $C M$ : mean square, $F$ : Fisher's statistic, $P$ : corresponding significance values).

\begin{tabular}{|c|c|c|c|c|}
\hline Fuente de variación & $G L$ & $C M$ & $F$ & $P$ \\
\hline \multicolumn{5}{|c|}{ Crecimiento de los brotes } \\
\hline Tratamiento & 1 & 78,76 & 12.1588 & 0,0030 \\
\hline Sitio & 1 & 0,02 & 0,0041 & 0,9497 \\
\hline Tratamiento $\mathrm{x}$ sitio & 1 & 0,50 & 0,07854 & 0,7829 \\
\hline Residual & 16 & 6,47 & & \\
\hline Total & 19 & 9,62 & & \\
\hline \multicolumn{5}{|c|}{ Peso específico foliar (PEF, g.m $\left.\mathrm{m}^{-2}\right)$} \\
\hline Tratamiento & 1 & 2,78 & 0,0139 & 0,9076 \\
\hline Sitio & 1 & 424,95 & 2,1309 & 0,1637 \\
\hline Tratamiento $\mathrm{x}$ sitio & 1 & 40,87 & 0,2049 & 0,6568 \\
\hline Residual & 16 & 199,22 & & \\
\hline Total & 19 & 192,60 & & \\
\hline
\end{tabular}




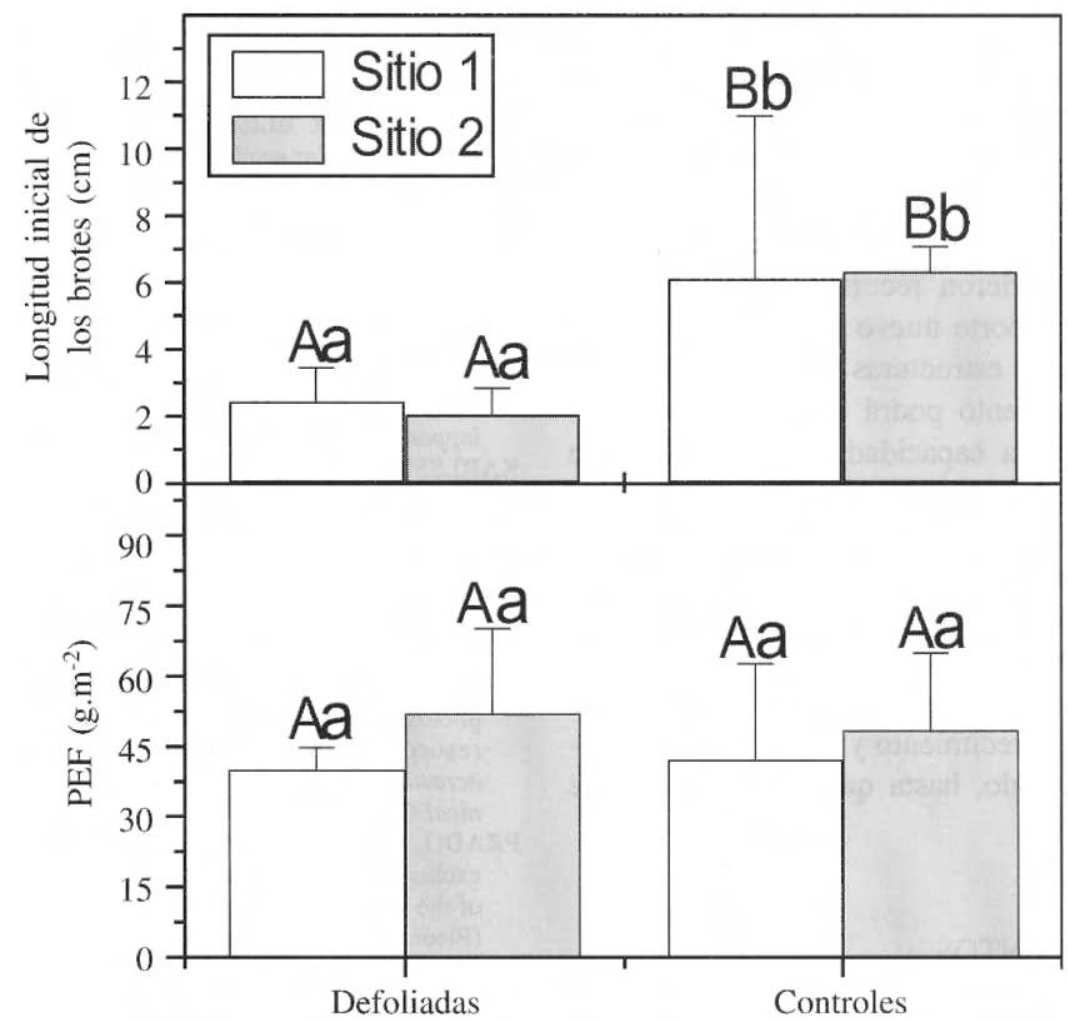

Figura 1. Valores medios (columnas) \pm desviación standard (barras) del crecimiento lineal inicial de los brotes y del peso específico foliar en individuos defoliados y controles de la especie inviernoverde Aristotelia chilensis en dos sitios del bosque de Nothofagus dombeyi. Distintas letras mayúsculas indican diferencias significativas $(\mathrm{p}<0,05)$ entre tratamientos. Las diferencias significativas de los valores medios de longitud de los brotes y peso específico foliar entre sitios son indicados con diferentes letras minúsculas.

Mean values (columns) \pm standard deviation (bars) of the initial linear branch growth and the specific leaf mass in defoliated and control individuals of the wintergreen species Aristotelia chilensis in two sites at Nothofagus dombeyi forest. Distinct capital letters indicate significant differences $(\mathrm{p}<0,05)$ between treatments. The significant differences of the mean values of branch length and specific leaf mass between sites are indicated by different low-case letters.

lance positivo de carbono en períodos menos favorables (Minoletti y Boerner, 1993; Jonasson, 1995). En el presente estudio se muestra además que la planta asegura la formación de sus órganos autotróficos independientemente de la actividad de las hojas viejas. En la figura 1 y en el cuadro 2 se observa que el peso específico foliar de la nueva cohorte foliar de primavera no difirió entre las plantas defoliadas y los controles, independientemente del sitio de muestreo. Esto indicaría que la cohorte de hojas nuevas se construye igual, posiblemente a expensas de un menor crecimiento de los brotes portadores. Estas nuevas hojas se ex- panden rápidamente y unos días antes de la senescencia de todas las hojas viejas tienen un tamaño igual al 95\% del de una hoja madura, aunque su PEF es menor (cuadro 1). Además las hojas nuevas cuando son sometidas a un alto flujo fotónico $\left(1500 \mathrm{~m} \mathrm{~mol} \mathrm{~m} \mathrm{~m}^{-2} \mathrm{~s}^{-1}\right)$ tienen una fotosíntesis expresada en área similar a la de las hojas viejas (Prado y Damascos, en prensa). La planta, entonces, asegura la formación de los órganos encargados de la intercepción de la luz durante el inicio de la estación de crecimiento, período en el que necesita carbono, y en las plantas defoliadas esto es inclusive necesario para compensar las 
pérdidas debidas a la ausencia de las hojas viejas en primavera. El balance de carbono más negativo debido a la eliminación de las hojas viejas puede ser verificado a través del crecimiento significativamente menor de los brotes de las plantas defoliadas (figura 1). Esto indicaría que las plantas defoliadas no tuvieron recursos suficientes para invertir en un soporte nuevo para incrementar el crecimiento de las estructuras vegetativas y reproductivas. Este evento podrá tener consecuencias negativas sobre la capacidad de reproducción e intercepción de la radiación de dichas plantas. Una forma de minimizar esta situación parecería ser: garantizar la inversión en la cohorte de hojas nuevas a principios de primavera, manteniendo el balance de carbono lo más positivo posible, lo cual permitirá a la planta suplir los requerimientos de recursos para el crecimiento y la reproducción durante dicho período, hasta que la nueva cohorte foliar se expanda.

\section{AGRADECIMIENTOS}

Agradecemos a los dos revisores anónimos que realizaron valiosas sugerencias y contribuyeron a mejorar la calidad del manuscrito.

\section{REFERENCIAS}

BARROS, V., V. CORDON, C. MOYANO, R. MENDEZ, J FORGUERA, O. PIZZIO. 1983. Cartas de precipitación de la zona oeste de las provincias de Río Negro y Neuquén. Primera Contribución. UNC, CONICET.
BAZZAZ, F.A., N.R. CHIARELLO, P.D. COLEY, L.F. PITELKA. 1987. "Allocating Resources to Reproduction and Defense", BioScience 37: 58-67.

BELL, K.L., L.D. BLISS. 1977. "Overwinter phenology of plants in a polar semidesert", Arctic 30: 118-121.

DAMASCOS, M.A. 1996. Análisis morfológico-estructural en comunidades de la Patagonia austral. Tesis doctoral. Universidad Nacional de La Plata, Argentina.

DAMASCOS, M.A. 1998. "Tendencias morfológicas de las plantas de los claros y áreas sombreadas del bosque de Austrocedrus chilensis, Argentina", Ecología Austral 8: 13-22.

JONASSON, S. 1995. "Resource allocation in relation to leaf retention time of the wintergreen Rhododendron lapponicum", Ecology 76: 475-485.

KARLSSON, P.S. 1994. The significance of internal nutrient cycling in branches for growth and reproduction of Rhododendron lapponicum, Oikos 70: 191-200.

LANDHÄUSSER, S.M., K.J. STADT, V.J. LIEFFERS. 1997. "Photosynthetic strategies of summergreen and evergreen understory herbs of the boreal mixedwood forest", Oecologia 112: $173-178$.

MINOLETTI, M.L., R.E.J. BOERNER. 1993. "Seasonal photosynthesis, nitrogen and phosphorus dynamics, and resorption in the wintergreen fern Polystichumacrostichoides (Michx) Schott", Bulletin of the Torrey Botanical Club 120: 397-404.

PRADO, C.H.B.A., M.A. DAMASCOS (en prensa). Gas exchange and leaf specific mass of different foliar cohorts of the wintergreen shrub Aristotelia chilensis (Mol.) Stuntz (Eleocarpaceae) fifteen days before the flowering and the fall of the old cohort. Brazilian Archives of Biology and Technology.

SNOW, A. A., D.F. WHIGHAM. 1989. "Cost of flower and fruit production in Tipularia discolor (Orchidaceae)", Ecology 70: 1286-1293.

SOKAL, R.R., F.J. ROHLF. 1981. Biometry. 2nd. ed., W. H. Freeman and Co., San Francisco, 361 pp.

TISSUE, D.T., J.B. SKILLMAN, E.P. MCDONALD, B.R. STRAIN. 1995. "Photosynthesis and carbon allocation in Tipularia discolor (Orchidaceae), a wintergreen understory herb", American Journal of Botany 82 : 1249-1256.

WESTOBY, M. 1998. "A leaf-height-seed (LHS) plant ecology strategy schema", Plant and Soil 199: 213-227.

WHIGHAM, D.F. 1990. "The effects of experimental defoliation on the growth and reproduction of a woodland orchid, Tipularia discolor", Canadian Journal of Botany 68: 1812-1816. 